\title{
Perfil epidemiológico das mulheres com síndromes hipertensivas na gestação e sua repercussão na prematuridade neonatal em uma maternidade pública de Belém/PA
}

\author{
Rhaysa Miranda Matias Dias*, Sara Negreiros Santos, M.Sc.**
}

\begin{abstract}
* Enfermeira graduada pelo Centro Universitário do Estado do Pará, Especialista em Enfermagem em Nefrologia pelo Instituto de Ensino, Formação e Aperfeiçoamento em Pós-Graduação de Patos/PB, Especialista em Enfermagem Oncológica pela Universidade Federal do Estado do Pará e Pós-graduanda pelo Programa de Residência Multiprofissional em Saúde especialista em Atençāo à Saúde da Mulher e da Criança pela Universidade Estadual do Pará e Fundação Santa Casa de Misericórdia do Pará (FSCMPA), ${ }^{* *}$ Enfermeira da FSCMPA, orientadora, preceptora da residência, Profa. da UEPA, Especialista em Obstetrícia
\end{abstract}

\section{Resumo}

Objetivo: Traçar o perfil epidemiológico de puérperas com Doença Hipertensiva Específica da Gravidez (DHEG), no ano de 2013, atendidas na Fundação Santa Casa de Misericórdia do Pará, Belém/PA, identificando os fatores de risco e correlacionando às repercussóes das síndromes com a prematuridade neonatal. Método: Estudo transversal retrospectivo, com abordagem quantitativa, com uma amostra de 200 puérperas que evoluíram com partos vaginal e cesárea de 205 RNs. A coleta de dados foi por meio da análise de prontuários. Resultados: A maioria das mulheres evoluiu para parto cesárea (94,5\%), era primigesta e solteira, seus RNs evoluíram com Idade Gestacional (AIG) (49,8\%), seguido Pequeno para a Idade Gestacional (PIG) (38,0\%), nasceram a termo (52,2\%), seguido de prematuros (27,3\%). Quanto ao tratamento, o mais frequente foi a terapêutica com Oxi-hood (39,8\%), seguido por entubaçáo orotraqueal (24,8\%). Conclusão: Evidenciou-se o número de RNs com baixo peso e prematuros que necessitou de suporte ventilatório e UTI, comprovando que as DHEG levam a complicaçóes imediatas e tardias nos neonatos.

Palavras-chave: Enfermagem, hipertensão, prematuridade, epidemiologia.

\section{Abstract}

Epidemiological profile of women with hypertensive syndrome in pregnancy and its impact on prematurity newborn in a public maternity of Belém/Pará, Brazil

Objective: To determine the epidemiological profile of mothers with preeclampsia (PE), in 2013, atended at Fundação Santa Casa de Misericórdia do Pará, Belém/PA, Brazil, and to identify risk factors and to correlate the impact of syndromes with neonatal prematurity. Method: Retrospective cross-sectional study with a quantitative approach, with a sample of 
200 mothers who had vaginal deliveries and cesarean section of 205 newborns. The medical record review was used as data collection. Results: Most women underwent cesarean section (94.5\%), were primigravid and single. The newborns were identified as followed: (49.8\%) appropriate for gestational age (AGA), (38.0\%) small for gestational age (SGA), (52.2\%) full term and (27.3\%) premature. Regarding the treatment, the most common therapy was Oxy-hood (39.8\%), followed by orotracheal intubation $(24.8 \%)$. Conclusion: It was noted that a large number of newborns with low birth weight and preterm infants required mechanical ventilation and ICU, which makes evident that the preeclampsia leads to immediate and later complications in newborns.

Key-words: Nursing, hypertension, prematurity, epidemiology.

\section{Resumen}

\section{Perfil epidemiológico de mujeres con síndromes hipertensivos en el embarazo y su impacto en la prematuridad neonatal en una maternidad pública de Belém/ Pará, Brasil}

Objetivo: Determinar el perfil epidemiológico de madres con Enfermedad Hipertensiva del Embarazo (EHE), en el año de 2013, que fueron atendidas en Fundação Santa Casa de Misericórdia do Pará, Belém/PA, Brasil, e identificar factores de riesgo y correlacionar el impacto de los síndromes con la prematuridad neonatal. Método: Estudio retrospectivo transversal con enfoque cuantitativo, con una muestra de 200 madres que tuvieron partos vaginales y cesáreos de 205 recién nacidos. La recolección de datos fue mediante revisión de historias clínicas. Resultados: La mayoría de las mujeres evolucionaron para el parto por cesárea $(94,5 \%)$, eran primigestas y solteras. Los recién nacidos $(\mathrm{RN})$ fueron identificados con edad gestacional adecuada (EGA) un 49,8\%, pequeño para la edad gestacional (PEG) un 38,0\%, recién nacido a término un $52,2 \%$ y prematuros un $27,3 \%$. En cuanto al tratamiento, el Oxy-hood ha sido la terapia más común $(39,8 \%)$, seguido por la intubación orotraqueal $(24,8 \%)$. Conclusión: Se evidenció el alto número de RN con bajo peso al nacer y prematuros que requirieron ventilación mecánica y UCI, comprobando que la EHE lleva a complicaciones inmediatas y tardías en los RN.

Palabras-clave: Enfermería, hipertensión, prematuridad, epidemiología.

\section{Introdução}

A gravidez é um processo natural e dinâmico que envolve diversas mudanças fisiológicas complexas, com evidências de muitos desafios durante esse período, como as síndromes hipertensivas específicas da gravidez [1]. Essas síndromes, ocorridas durante o período gravídico puerperal, são as complicaçóes obstétricas de maior relevância, com incidência de $6 \%$ a $30 \%$ das gestantes, resultando em alto risco de morbimortalidade materna e perinatal [1-3].

As duas principais causas específicas de morte materna no Brasil são as Síndromes Hipertensivas da gestação e a Hemorragia de modo geral. No Brasil, entre os anos de 1990 e 2010, as alteraçóes no padrão de causas específicas de morte materna mostram uma redução de $66,0 \%$ no risco de morrer por hipertensão $(1990-40,6 \%, 2000-21,5 \%$, 2010-13,8\%); de 69,3\% por hemorragia; de 60,4\% por infecção puerperal; de $81,9 \%$ por aborto; e de $42,5 \%$ por doenças do aparelho circulatório que complicam a gravidez, o parto e o puerpério. Nota- -se que as quedas de risco mais evidentes ocorreram entre as mortes por aborto e por hemorragia [4].

A falta de assistência nas pacientes com síndromes hipertensivas ou a sua evoluçáo desfavorável pode levar ao óbito, o que faz dessa doença a responsável pela mortalidade materna nos países da América Latina e Caribe, incluindo o Brasil [5].

A Doença Hipertensiva Específica da Gravidez (DHEG) apresenta potencialmente os piores prognósticos materno-fetal, sendo assim, conceptos de mães hipertensas têm maiores riscos de prematuridade, ocorrência de partos de fetos pequenos para idade gestacional (PIG), necessidade de Unidade de Tratamento Intensivo (UTI) neonatal, necessidade de suporte ventilatório e maior incidência da mortalidade perinatal. Além disso, pode cursar com encefalopatia hipóxico-isquêmica ao concepto levando a subsequentes manifestaçôes neurológicas [6,7].

Neste contexto, destaca-se o importante papel da sistematização da assistência de enfermagem direcionada as síndromes hipertensivas uma vez que a documentação empregada na consecução do 
processo de enfermagem garantiria a identificação das pacientes possibilitando desta forma desenhar o perfil epidemiológico das gestantes, ao mesmo tempo em que possibilitaria a elaboração do plano assistencial individualizado [2].

Diante da magnitude dessa patologia e do risco obstétrico e neonatal que representa para mãe e filho motivou a realização deste presente estudo, que tem como objetivos: traçar o perfil epidemiológico de mulheres com Síndromes Hipertensivas da gestação do ano de 2013 de uma maternidade pública de Belém correlacionando às repercussóes delas com prematuridade neonatal.

\section{Material e métodos}

Trata-se de um estudo com abordagem quantitativa de caráter transversal e retrospectivo. A coleta de dados foi realizada por meio de pesquisa aos prontuários das puérperas e de seus recém-nascidos. Foi utilizado um formulário padronizado no qual foram coletadas informaçóes acerca dos dados sociodemográficos das mulheres, seus antecedentes pessoais, dados obstétricos e dados relacionados ao nascimento dos bebês. Para realização da pesquisa utilizou-se como base os dados disponíveis no setor de Serviço de Arquivo Médico Estatístico da instituição.

O projeto de pesquisa foi submetido ao Comitê de Ética e Pesquisa (CEP) da Fundação Santa Casa de Misericórdia do Pará, em Belém/PA, e aprovado sob o parecer $n^{\circ}$ XXX, conforme a resoluçáo $466 / 12$ do Conselho Nacional de Saúde.

A amostra constitui-se de 200 puérperas que evoluíram com parto vaginal e cesárea e de 205 recém-nascidos, de julho a dezembro de 2013. Estabeleceu-se como critérios de inclusão puérperas com algum diagnóstico de DHEG e seus recém-nascidos, primigestas ou multigestas, parto de concepto com idade gestacional acima de 20 semanas, gestação única e ou gemelar e idade materna acima de 14 anos.

Os dados obtidos foram transferidos para uma planilha e tabulados com o auxílio dos programas Microsoft Excel e Microsoft Word versões do Office 2007. Para a confecção das tabelas e figuras, utilizou-se de números absolutos e porcentagens.

\section{Resultados e discussão}

Em relação aos dados epidemiológicos maternos da amostra pesquisada houve uma prevalência na idade entre 14 e 47 anos, assim como foi obser- vado por Oliveira et al. [9], no seu estudo sobre os fatores de risco para mortalidade materna de puérperas em um hospital público de São Paulo onde a maioria $(57,0 \%)$ estava na faixa etária de 15 a 46 anos. A faixa etária prevalente evidencia que o maior predomínio de casos de DHEG ocorreu na fase com maior probabilidade de engravidar e não nos extremos da fase procriativa. Em relação à escolaridade, mais da metade das puérperas possuíam o ensino médio $(55,3 \%)$. Dados próximos aos encontrados por Frigo et al. [10], em que 66,6\% apresentavam ensino médio e apenas 33,3\% mulheres com ensino fundamental completo.

O estado civil das puérperas pesquisadas mostrou que a maioria $(49,7 \%)$ era solteira. De acordo com Gonçalves, Fernandes e Sobral [2], o perfil de 122 puérperas com DHEG na cidade de Ceará em um hospital foi em maior proporção de solteiras. Este quesito é considerado imprescindível para a uma boa evolução da gestação, pois, segundo alguns autores, o papel paterno é essencial como apoio.

Tabela I - Distribuição das puérperas que tiveram um dos diagnósticos de DHEG e de seus recém-nascidos atendidos na maternidade do Hospital de Referência Materno-Infantil de Belém/PA no período de julho a dezembro de 2013, de acordo com as características epidemiológicas maternas.

\begin{tabular}{|c|c|c|}
\hline $\begin{array}{c}\text { Características epidemiológicas } \\
\text { maternas }\end{array}$ & $N$ & $\%$ \\
\hline \multicolumn{3}{|l|}{ Idade da mãe (em anos) } \\
\hline Até 18 & 34 & 17,1 \\
\hline 19 a 25 & 76 & 38,2 \\
\hline 26 a 30 & 45 & 22,6 \\
\hline 31 a 35 & 28 & 14,1 \\
\hline Acima de 35 & 17 & 8,0 \\
\hline Total & 200 & 100,0 \\
\hline \multicolumn{3}{|l|}{ Estado civil } \\
\hline Casada & 28 & 14,1 \\
\hline Divorciada & 1 & 0,5 \\
\hline Solteira & 99 & 49,7 \\
\hline União estável & 72 & 35,7 \\
\hline Total & 200 & 100,0 \\
\hline \multicolumn{3}{|l|}{ Escolaridade } \\
\hline Sem escolaridade & 24 & 12,0 \\
\hline Ensino fundamental & 62 & 30,7 \\
\hline Ensino médio & 110 & 55,3 \\
\hline Ensino superior & 4 & 2,0 \\
\hline Total & 200 & 100,0 \\
\hline
\end{tabular}

FONTE: Dados coletados na pesquisa. 
Em relação aos fatores de risco da amostra pesquisada houve uma prevalência na realização do pré-natal (89,9\%). Calderon et al. [11], em uma revisão de literatura deixa clara a importância da realização de pré-natal para potenciais riscos, destacando as intervençóes específicas relacionadas a promoção da saúde materna, prevenção dos riscos e garantia de suporte nutricional durante a gestação, além de critérios para investigação do risco gestacional e inclusão da gestante no programa de imunizaçáo materna e controle de doenças.

Nesta pesquisa a maioria das mães que realizou o pré-natal 179 (89,9\%) apresentou uma variável positiva para o perfil epidemiológico. Demonstrando através desses dados uma cobertura da assistência ao pré-natal dessa populaçáo em risco obstétrico; porém essa variável deixa de ser favorável na sua totalidade devido ao elevado índice de mulheres com DHEG evoluindo para partos prematuros com nascimentos de recém-nascidos de baixo peso evidenciando assim falhas na qualidade da assistência. Mais da metade possuía antecedentes familiares $(65,3 \%)$, semelhante à pesquisa no estado do Pará que aponta como casuística clínica encontrada a Hipertensão Arterial Sistêmica (HAS) que aparece como maioria com $(63,3 \%)$ seguida de DM (36.7\%). Sabe-se que o perfil epidemiológico da DHEG, uma vez que é conhecido, sua incidência clínico-obstétrica é cerca de quatro vezes maior em filhas de mães que já tiveram a doença, comparadas à população $[12,13]$.

Observou-se que apenas $10,1 \%$ das mães eram tabagistas, 4,5\% faziam uso de drogas ilícitas e $14,1 \%$ uso de álcool. No que concerne à descrição de vícios nocivos durante a gravidez/puerpério, o Ministério da Saúde recomenda que os cuidados no pré-natal devam ser somados à vigilância rigorosa do crescimento fetal e de sua vitalidade [4].

Mais da metade era primigesta $(57,3 \%)$, semelhante ao estudo Plasencia et al. em que a avaliação das características obstétricas mostrou que quase $65 \%$ das pacientes eram primigestas, o que diverge dos mais de $40 \%$ encontrados por outros autores. A exclusão de pacientes fora do trabalho de parto e com passado de hipertensão crônica parece direcionar a seleção para pacientes mais jovens e primigestas [14].

Identificou-se que no presente estudo a maioria das mulheres $(94,5 \%)$ evoluiu para parto cesáreo, corroborando o estudo de Martins, Rezende e Vinhas, no qual $(82,85 \%)$ das gestantes com DHEG evoluíram para o parto cesáreo. A frequência de quase $80 \%$ de cesarianas está bem acima do recomendado pela Organizaçáo Mundial de Saúde [15].

Quanto aos antecedentes pessoais, o mais frequente foi a HAS presente em $81,4 \%$ das mães. Confirmando os achados da literatura ao dizerem que as chances de uma mulher desenvolver pré-eclâmpsia são $25 \%$ maior quando apresentar história de hipertensão arterial pregressa [16].

A Infecção do Trato Urinário (ITU) foi a intercorrência mais frequente, presente em 50,3\% das mães, semelhante ao estudo de Alves, quanto à frequência das patologias maternas, durante o período gravídico das puérperas, constatou-se que 49,2\% (30) referiram infecção urinária [17].

$\mathrm{O}$ diagnóstico mais frequente entre as puérperas foi Pré-Eclâmpsia (presente em 50,2\% delas). Segundo Rezende [18], em primíparas, a maior parte (2/3) dos casos de pré-eclampsia é mais frequente no $\mathrm{RN}$ a termo, e a maioria dos $\mathrm{RN}$ tem crescimento normal e saudável. Em multíparas parece ser diferente uma vez que a pré-eclampsia incide em população de alto-risco e o tipo de doença é geralmente precoce-grave (antes de 34 semanas). No estudo de Oliveira et al., metade das mulheres tiveram pré-eclampsia, e 45,6\% dos seus RNs evoluíram com baixo peso [6].

Tabela II - Distribuição das puérperas que tiveram um dos diagnósticos de DHEG e de seus recém-nascidos atendidos na maternidade do Hospital de Referência Materno-Infantil de Belém/PA no período de julho a dezembro de 2013, de acordo com os fatores de risco materno.

\begin{tabular}{|c|c|c|}
\hline Fatores de risco & $\mathrm{N}$ & $\%$ \\
\hline \multicolumn{3}{|l|}{ Pré-natal } \\
\hline Não & 21 & 10,1 \\
\hline Sim & 179 & 89,9 \\
\hline Total & 200 & 100,0 \\
\hline \multicolumn{3}{|l|}{ Antecedentes familiares } \\
\hline Não & 69 & 34,7 \\
\hline Sim & 131 & 65,3 \\
\hline Total & 200 & 100 \\
\hline \multicolumn{3}{|c|}{ Tabagismo } \\
\hline Não & 181 & 89,9 \\
\hline Sim & 19 & 10,1 \\
\hline Total & 200 & 100,0 \\
\hline \multicolumn{3}{|l|}{ Drogas ilícitas } \\
\hline Não & 190 & 95,5 \\
\hline Sim & 10 & 4,5 \\
\hline Total & 200 & 100,0 \\
\hline
\end{tabular}




\begin{tabular}{lcc}
\hline Primigesta & & \\
\hline Não & 86 & 42,7 \\
$\mathrm{Sim}$ & 114 & 57,3 \\
Total & 200 & 100,0 \\
\hline Multigestas & & \\
\hline Não & 116 & 58,3 \\
Sim & 84 & 41,7 \\
Total & 200 & 100,0 \\
\hline Etilismo & & \\
\hline Não & 171 & 85,9 \\
Sim & 29 & 14,1 \\
Total & 200 & 100,0 \\
\hline Intercorrências & & \\
\hline Não & 110 & 54,8 \\
Sim & 90 & 45,2 \\
Total & 200 & 100,0 \\
\hline Tipo de parto & & \\
Cesárea & 188 & 94,5 \\
Normal & 12 & 5,5 \\
Total & 200 & 100,0 \\
\hline FON & & \\
\hline
\end{tabular}

FONTE: Dados coletados na pesquisa.

Tabela III - Distribuição das puérperas que tiveram um dos diagnósticos de DHEG e de seus recém-nascidos atendidos na maternidade do Hospital de Referência Materno-Infantil de Belém/PA no período de julho a dezembro de 2013, de acordo com os antecedentes familiares mais frequentes.

\begin{tabular}{lcc}
\hline $\begin{array}{c}\text { Antecedentes familiares } \\
\text { mais frequentes }\end{array}$ & N & $\begin{array}{c}\text { \% relativo aos } \\
\text { 131 pacientes }\end{array}$ \\
\hline HAS & 107 & 50,5 \\
DM & 13 & 30,0 \\
Gemelar & 6 & 19,0 \\
Cardiopata & 5 & 0,5 \\
Total & 131 & 100 \\
\hline
\end{tabular}

FONTE: Dados coletados na pesquisa.

Tabela IV - Distribuição das puérperas que tiveram um dos diagnósticos de DHEG e de seus recém-nascidos atendidos na maternidade do Hospital de Referência Materno-Infantil de Belém/PA no período de julho a dezembro de 2013, de acordo com os antecedentes pessoais mais frequentes.

\begin{tabular}{lcc}
\hline $\begin{array}{c}\text { Antecedentes } \\
\text { pessoais mais } \\
\text { frequentes }\end{array}$ & $\mathrm{N}$ & $\begin{array}{c}\text { \% relativo aos 59 pa- } \\
\text { cientes com anteceden- } \\
\text { tes pessoais }\end{array}$ \\
\hline HAS & 107 & 81,4 \\
DM & 13 & 18,6 \\
\hline Total & 120 & 100 \\
\hline
\end{tabular}

FONTE: Dados coletados na pesquisa.
Figura I - Distribuição das puérperas que tiveram um dos diagnósticos de DHEG e de seus recém-nascidos atendidos na maternidade do Hospital de Referência Materno-Infantil de Belém/PA no período de julho a dezembro de 2013, de acordo com as intercorrências sofridas.

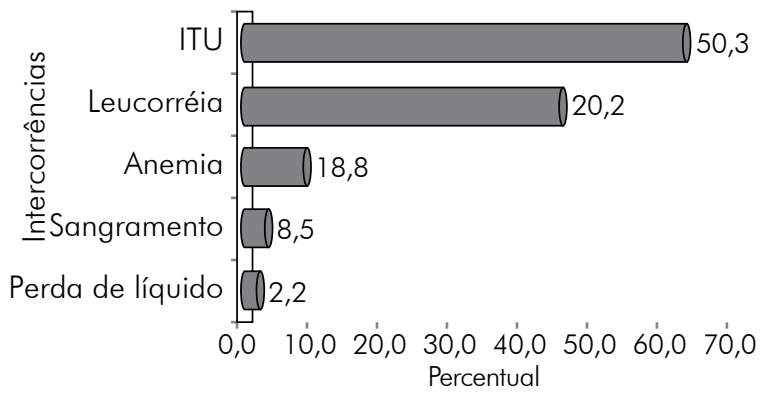

Tabela $\mathbf{V}$ - Distribuição das puérperas que tiveram um dos diagnósticos de DHEG e de seus recém-nascidos atendidos na maternidade do Hospital de Referência Materno-Infantil de Belém/PA no período de julho a dezembro de 2013, de acordo com os diagnósticos.

\begin{tabular}{lcc}
\hline $\begin{array}{c}\text { Diagnósticos mais fre- } \\
\text { quentes nas mães }\end{array}$ & $\mathrm{N}$ & $\begin{array}{c}\text { \% relativo ao total } \\
\text { de mães }\end{array}$ \\
\hline Pré-eclâmpsia & 68 & 50,2 \\
Eclâmpsia & 50 & 19,1 \\
DHEG grave & 45 & 15,2 \\
DHEG leve & 20 & 9 \\
Iminência de eclampsia & 11 & 5,2 \\
Síndrome de Hellp & 5 & 1,1 \\
DHEG superjuntada & 1 & 0,2 \\
\hline TOTAL & 200 & 100 \\
\hline
\end{tabular}

FONTE: Dados coletados na pesquisa.

Verifica-se que o peso mais frequente dos RNs foi AIG (49,8\%), seguido pela categoria (PIG). A idade gestacional dos bebês mais frequente foi a termo $(52,2 \%)$, seguido pelos que nasceram prematuros $(27,3 \%)$. $\mathrm{O}$ peso ao nascimento é considerado como sendo um dos principais fatores a determinar a probabilidade de sobreviver ao período neonatal e ao mesmo tempo o restante do primeiro ano de vida [19].

No estudo de Oliveira e Chaim, observaram-se resultados semelhantes, no qual $(68,1 \%)$ dos RNs eram adequados para idade gestacional. No estudo de Sibai et al., nas gestantes que apresentaram pré-eclampsia, a maioria teve recém-nascidos considerados pequenos para idade gestacional. Para a Sociedade Australiana de Estudo de Hipertensão na Gravidez, cerca de 25\% dos filhos 
de máes com pré-eclampsia são pequenos para a idade gestacional $[9,16,20]$.

A prematuridade aumenta a taxa de morbidade e mortalidade perinatal com possibilidades de sequelas imediatas ou tardias. Constituindo uma das complicaçóes mais frequentes da DHEG, decorrente de trabalho de parto espontâneo, em razão da contratilidade uterina aumentada. Comumente a conduta obstétrica é de interrupção da gravidez $[21,22]$.

Verificou-se que 3,4\% das crianças tiveram má formação, houve desconforto respiratório em mais da metade (52,2\%). Quanto ao tratamento, $66,3 \%$ realizaram algum tipo, sendo mais frequente a terapêutica com Oxi-hood (39,8\%), seguido por entubação orotraqueal $(24,8 \%)$, a indicação médica mais frequente foi ALCON $(55,6 \%)$ seguida de unidade neonatal (berçário- 33,7\%) e UTI (9,3\%). No estudo de Alves, em um hospital do Rio Grande Sul evidenciou-se que os fatores que contribuíram para precisão de cuidados neonatais na UTI de RNs filhos de puérperas com DHEG foi baixo peso (47.9\%), a prematuridade com $(39,3 \%)$, a disfunção respiratória com $(19,3 \%)$ e o Sofrimento fetal agudo (SFA) $(13,1 \%)$ [18]. Tal fato corrobora o estudo Dias, quanto a dificuldade respiratória, em que 43,3\% dos RNs necessitaram de $\mathrm{O} 2$ e $66,7 \%$ dos recém-nascidos filhos de mães hipertensas necessitaram de tratamentos mais invasivos como oxi-hood e ventilação mecânica invasiva [8].

Tabela VI - Distribuição das puérperas que tiveram um dos diagnósticos de DHEG e de seus recém-nascidos atendidos na maternidade do Hospital de Referência Materno-Infantil de Belém/PA no período de julho a dezembro de 2013, de acordo com as características dos RNs.

\begin{tabular}{lcc}
\hline \multicolumn{1}{c}{ Características do recém-nascido } & $\mathrm{N}$ & $\%$ \\
\hline Peso (classificação) & & \\
\hline Sem informação & 1 & 0,5 \\
AIG & 102 & 49,8 \\
GIG & 24 & 11,7 \\
PIG & 78 & 38 \\
Total & 205 & 100 \\
\hline Idade gestacional & & \\
\hline Prematuro & 56 & 27,3 \\
Termo & 141 & 52,2 \\
Pós-termo & 5 & 19 \\
Não informado & 3 & 1,5 \\
Total & 205 & 100 \\
\hline Má formaçãolCromossopatia & & \\
\hline Não informado & 3 & 1,5
\end{tabular}

\begin{tabular}{lcc} 
Não & 195 & 95,1 \\
Sim & 7 & 3,4 \\
Total & 205 & 100 \\
\hline Desconforto respiratório & & \\
\hline Não informado & 3 & 1,5 \\
Não & 95 & 46,3 \\
Sim & 107 & 52,2 \\
Total & 205 & 100 \\
\hline Tratamento & & \\
\hline Não & 69 & 33,7 \\
Sim & 136 & 66,3 \\
Total & 205 & 100 \\
\hline Indicação médica & & \\
\hline ALCON & 109 & 55,6 \\
Berçário & 69 & 33,7 \\
UTI & 19 & 9,3 \\
Não informado & 3 & 1,4 \\
\hline Total & 205 & 100 \\
\hline FONTE Dados coltados na pesqusa & &
\end{tabular}

FONTE: Dados coletados na pesquisa.

\section{Conclusão}

Conclui-se que os principais fatores de risco para evoluçáo da DHEG neste estudo foram idade na faixa etária de 19 a 25 anos, prevalência de puérperas solteiras, a baixa escolaridade, ser primigesta, antecedentes familiares e pessoais como HAS e DM e a falta de qualidade na assistência ao pré-natal.

Pode-se concluir que essas síndromes levam a complicaçóes no parto como: descolamento prematuro de placenta, o sofrimento fetal agudo e partos prematuros. A análise dos resultados mostrou que o número de recém-nascidos com baixo peso e que necessitou de suporte ventilatório foi evidente. Sendo assim, comprova-se que as síndromes hipertensivas específicas da gestação levam a complicações imediatas nos neonatos, podendo haver complicaçóes tardias.

Observou-se o quanto os recém-nascidos de mães com síndromes hipertensivas necessitam de inúmeros tratamentos especializados que visam um bom acompanhamento dos bebês dentro da UTI e berçário principalmente quando os mesmos nascem prematuros e com baixo peso. Fatores estes que geram várias repercussốes na vida dos $\mathrm{RN}$, impedindo, muitas das vezes, a alta hospitalar no período favorável de 48 horas como estabelecido pelo Ministério da Saúde.

Os resultados encontrados apontam também para a ausência de registros fundamentais e/ou preenchimento insuficiente de diversos dados no prontuário, sendo uma constante observada du- 
rante toda a coleta, isso se pode observar através da leitura das tabelas e dos gráficos, o que dificulta a realização de pesquisas para a caracterização da população estudada. Nesse sentido, é fundamental que os enfermeiros participem e envolvam-se em estratégias voltadas à vigilância epidemiológica para melhoria dos indicadores que envolvem a DHEG.

\section{Referências}

1. França CM. A prevalência de gestantes portadoras de DHEG que apresentam a síndrome de HELLP em uma maternidade de referência em Maceió/AL. Projeto de pesquisa da Universidade Estadual de Ciências da Saúde de Alagoas (UNCISAL). Trabalho 197 apresentado no VII Congresso Brasileiro de Enfermagem Obstétrico e neonatal, 2011;1:1943-77.

2. Gonçalves R, Fernandes RA, Sobral DH. Prevalência da doença hipertensiva específica da gestação em um hospital público de São Paulo. Rev Bras Enferm 2005;58:61-4.

3. Assis TR, Viana, FP, Rassi S. Estudos dos principais fatores de risco maternos nas síndromes hipertensivas da gestação. Arq Bras Cardiol 2008;91:11-7.

4. Ministério da Saúde. Boletim Epidemiológico 1/2012. Mortalidade materna no Brasil. Volume $43^{\circ}$. Secretaria de Vigilância em Saúde. Brasília: Ministério da Saúde; 2012.

5. Melo BC, Amorim MM, Katz L, Coutinho I, Verissimo G. Perfil epidemiológico e evoluçáo pós-parto na pré-eclampsia. Rev Assoc Med Bras 2009;29:135-47.

6. Oliveira CA, Lins CP, Sá RAM, Netto HC, Bornia RG, Silva NR. Síndromes hipertensivas da gestação e repercussōes perinatais. Rev Bras Saúde Mater Infant 2006;6:93-6.

7. Dias BR, Piovesana AMSG, Montenegro MA, Guerreiro MM. Avaliação da idade gestacional de recém-nascidos pré-termo através do exame neurológico e das escalas neonatais e obstétricas. Arq Neuropsiquiatr 2005;63:632-6.

8. Dias BR, Piovenasa AM, Montenegro MA, Guerreiro MM. Desenvolvimento neuropsicomotor de lactentes filhos e mães que apresentam hipertensão arterial na gestação. Arq Neuropsiquiatr 2012;70:84-100.
9. Oliveira MI, Vasconcelos SG, Puérperas com Síndrome de Hellp: análise baseada nos aspectos obstétricos. Rev Bras Ginecol Obstet 2011;10:28-36.

10. Frigo J, Bringhenti LM, Gollo AA, Ascari RA, Kolhs M., Marin, SM. Perfil epidemiológico de gestantes com doença hipertensiva específica da gestação atendidas no serviço de referência municipal. Enferm Foco 2013;4(2):109-11.

11. Calderon IMP, Cecatti JG, Veja CEP. Intervençôes benéficas no pré-natal para prevenção da mortalidade Materna. RBGO 2010;28:310-15.

12. Guerreiro DD. Mortalidade materna relacionada a DHEG em uma maternidade do Estado do Pará [monografia]. Belém: UEPA; 2014.

13. Melo BC, Amorim MM, Katz, Coutinho I, Verissimo G. Perfil epidemiológico e evolução pós-parto na pré-eclampsia. Rev Assoc Med Bras 2009;55:175-80.

14. Plasencia W, Maiz NS, Bonino KC, Nicolaides KH. Artéria uterina Doppler em $11+0-13+6$ semanas na predição de pré-eclâmpsia. Ultrasound Obstet Gynecol 2010;30:742-9.

15. Martins CA, Rezende LPR, Vinhas DCS. Gestação de alto risco e baixo peso ao nascer Goiânia. Rev Eletr Enferm 2009;5:45-9.

16. Chaim SRP, Oliveira SMJV, Kimura AF. Hipertensão arterial na gestaçáo e condiçóes neonatais ao nascimento. Acta Paul Enferm 2008;21:53-8.

17. Alves AS. Recém-nascidos de risco: fatores que contribuem para precisão de cuidados intensivos neonatais [Dissertaçáo]. Porto Alegre: UFRG; 2009.

18. Rezende J, Montenegro CA. Obstetrícia fundamental: toxemia gravídica pré-eclampsia e eclampsia. $12^{\mathrm{a}}$. ed. Rio de Janeiro: Guanabara Koogan; 2012.

19. Brasil. Ministério da Saúde. Pré-natal, puerpério e atençáo qualificada e humanizada. Brasília: MS; 2006.

20. SIBAI BM. Diagnosis and management of gestacional hypertension and preeclampsia. Obstet Gynecol 2003;102:181-92.

21. Coelho TM, Martins MG, Viana E, Mesquita MRS, Camano L, Sass N. Proteinúria nas síndromes hipertensivas da gestaçáo: prognóstico materno e perinatal. Rev Assoc Med Bras 2012;50:207-13.

22. Amorin MR, Faúndes A, Santos LC, Azevedo E. Acurácia do teste de Clements para avaliação da maturidade pulmonar fetal em gestantes com doença hipertensiva específica da gestação. Rev Bras Ginecol Obstet 1998;20:253-60. 